\title{
Intimate partner violence: How should health systems respond?
}

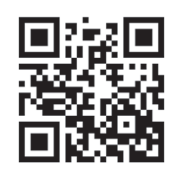

Intimate partner violence (IPV) is a common and devastating feature of partnerships worldwide, and South Africa (SA) is no exception. In 2013, the publication of World Health Organization (WHO) clinical and policy guidelines for responding to IPV and sexual violence ${ }^{[1]}$ supported the growing acknowledgement that IPV should be viewed as an issue directly related to health, and outlined an appropriate response from policy makers and healthcare providers. IPV is important to health systems because of the wide-ranging and serious health effects experienced by exposed women, as well as the unique opportunity afforded to healthcare providers to enquire about violence. IPV and gender inequality are also features of the complex network of causes driving the HIV epidemic in sub-Saharan Africa. In SA, where there is an extremely high burden of interpersonal violence, gender-based violence and HIV, IPV should be treated as a priority health concern.

For health services, one of the major implications is that healthcare providers should be supported in enquiring about and responding to IPV during healthcare encounters. There is sufficient evidence that intervening for IPV in a primary healthcare setting can be beneficial. A recent systematic review of interventions found that $76 \%$ of 17 included studies showed reductions in IPV or improvement in other health outcomes. ${ }^{[2]}$

Universal screening for IPV is controversial, although the need to identify cases non-routinely in healthcare settings is widely accepted. The US Preventive Services Task Force has recommended universal screening for IPV in women of childbearing age since 2013. ${ }^{[3]}$ However, more recently a rigorously conducted randomised controlled trial (the WEAVE study) found no difference in primary outcomes between women who were routinely screened for violence and a control group. ${ }^{[4]}$ This trial, in addition to prior evidence, ${ }^{[5,6]}$ has led to the expert conclusion that universal screening for IPV is ineffective in improving health. ${ }^{[7}$ Although screening is able to identify women experiencing IPV, uptake of interventions is impeded by numerous barriers and is often low, and current intervention approaches have not yet proved beneficial in asymptomatic women.

Enquiring about and discussing violence in specific cases during healthcare encounters (case finding) has been recommended as an alternative approach, ${ }^{[7,8]}$ followed by more complex, individualised interventions. This approach has been demonstrated to be feasible, with a cluster randomised controlled trial showing that training and support can significantly increase the number of women identified and referred to services in the absence of universal screening. ${ }^{[9]}$

Women who have experienced IPV have consistently described an appropriate response by healthcare providers to be nonjudgemental, understanding and empathetic. ${ }^{[10]}$ Women want their healthcare providers to understand the complexities and consequences of living with violence, and the difficulties they face because of it. ${ }^{[11]}$ They also want an acknowledgement from their providers that what they are experiencing is abuse, and that it is unacceptable and wrong. ${ }^{[12]}$

These features are highlighted in the WHO guidelines, which recommend that healthcare providers enquire about IPV when it may be relevant, and then provide women-centred care by maintaining confidentiality, supporting women in a non-judgemental manner, asking about a history of violence, and helping women to access information and increase their safety. ${ }^{[1]}$ Examples of encounters dur- 
ing which IPV should be raised include those concerning symptoms of common mental health disorders, alcohol and substance abuse, chronic pain or other unexplained symptoms, sexually transmitted infections and pregnancy or family planning.

Overall, the appropriateness of the healthcare encounter depends on the empathetic and non-directive attitude of the provider, the attention paid to emotional issues and the maintenance of confidentiality. If these elements are present, women experiencing IPV usually view the issue of violence being raised by a healthcare provider as supportive and helpful.

Despite the knowledge that healthcare provider intervention in IPV can be of benefit to patients, the evidence base informing the scaleup of IPV interventions and their integration into health systems is lacking. ${ }^{[13]}$ IPV interventions are complex, and require collaboration between many sectors such as health, social services and criminal justice, as well as systems that facilitate these. They therefore require more than provider training to enable effective programme functioning within a health system.

Examples of published investigations do, however, provide lessons of interest to those wishing to institute an appropriate response to IPV. In Malaysia, the national scale-up of One Stop Crisis Centres, an integrated health sector response to IPV, was investigated. Factors relating to health system structure and organisation, as well as external policy constraints, were found to be barriers to implementation. ${ }^{[13]}$ Several system-level factors arising from this case study could be applicable in other contexts. Commitment at policy level was found to be necessary, which could be communicated to service delivery level by incorporating appropriate indicators into routine reporting. Adequate training, as well as adjustments to service delivery to ensure that providers had the necessary time and privacy available to them, was required. Finally, flexibility of the model was important to allow its implementation at different levels of care.

An investigation of the integration of gender-based violence laws into the regional health systems of Spain found institutionalisation to be a challenge. ${ }^{[14]}$ Advances were often made through the actions of highly motivated individuals, raising concerns about sustainability. Budget allocation was found to be a key component of institutionalising change. It was also noted that since IPV is complex to respond to, protocols, while necessary, were insufficient and need to be supported by adequate training. ${ }^{[14]}$

In SA, Vezimfilho, a model health sector response to IPV, was developed and implemented in four districts. ${ }^{[15]}$ Important findings from an evaluation of the implementation process included the need for a systemic response, with political commitment, policies, protocols and effective referral systems being essential. ${ }^{[15]}$ In addition, training and capacity building needed to include addressing values and attitudes towards IPV and gender norms, as well as interpersonal skills in healthcare providers. Support from managers in the health system and strong relationships between multiple stakeholders were found to be key to a sustainable approach. ${ }^{[15]}$ System-level barriers to implementation included insufficient staff and lack of confidence in managerial support, while on a societal level providers' attitudes and perceptions relating to gender hampered implementation. ${ }^{[15]}$ The societal barriers relating to gender imply that a comprehensive health sector response requires advocating for wider social change.

In conclusion, there is sufficient evidence that IPV is a pervasive, serious public health concern, and that addressing IPV in health services has the potential to improve outcomes. Furthermore, in countries such as SA, addressing IPV and gender inequality should form an integral part of HIV prevention programmes.
For policy makers, there is an urgent need for policies and protocols that clearly frame IPV as an important health issue and support healthcare providers in enquiring about and responding to IPV. There is also a need for comprehensive undergraduate and in-service training, both allowing intervention for IPV to be viewed as an integral part of a healthcare provider's job, and imparting the knowledge and skills to enable this.

Individual healthcare providers should familiarise themselves with the content of the WHO clinical and policy guidelines for responding to IPV and sexual violence against women. They should enquire about IPV, support women who have disclosed violence in a nonjudgemental manner, and encourage their management structures to develop protocols if these do not exist.

For researchers, there is a need for the development and evaluation of health sector responses to IPV, to assist health systems in determining the most appropriate models of care, and how these can be integrated into current systems, in the context of multiple systemic and societal barriers. Further research is needed to explore how best to support health services in providing IPV care, how to operationalise intersectoral approaches to IPV in health systems, and how to improve access to, including acceptability of, services. The need for this research should not prevent health systems from implementing IPV care, but should guide the development of rigorous, contextually appropriate evaluations.

\section{Kate Rees}

Virginia Zweigenthal

Division of Public Health, Faculty of Health Sciences, University of Cape Town, South Africa

\section{Kate Joyner}

Nursing Division, Faculty of Medicine and Health Sciences, Stellenbosch University, Tygerberg, Cape Town, South Africa

\section{Corresponding author: K Rees (reeskste@gmail.com)}

1. World Health Organization. Responding to Intimate Partner and Sexual Violence Against Women: WHO Clinical and Policy Guidelines. Geneva: WHO, 2013. http://www.who.int/reproductivehealth/publications/ violence/9789241548595/en/ (accessed 28 May 2014)

Bair-Merritt MH, Lewis-O'Connor A, Goel S, et al. Primary care-based interventions for intimate partner violence: A systematic review. Am J Prev Med 2014;46(2):188-194. [http://dx.doi.org/10.1016/j.

Tor intimate pritner violence and abuse of elderly and vulnerable adults: Us Preventive Services Task Force recommendation statement. Ann Intern Med 2013;158(6):478-486. [http:// dx.doi.org/10.7326/0003-4819-158-6-201303190-00588]

4. Hegarty K, O'Doherty L, Taft A, et al. Screening and counselling in the primary care setting for women who have experienced intimate partner violence (WEAVE): A cluster randomised controlled trial. Lancet 2013;382(9888):249-258. [http://dx.doi.org/10.1016/S0140-6736(13)60052-5]

5. Klevens J, Kee R, Triack W, et al. Effect of screening for partner violence on women's quality of life: A randomized controlled trial. JAMA 2012;308(7):681-689. [http://dx.doi.org/10.1001/ jama.2012.6434]

Wathen $\mathrm{C}$, Jamieson E, et al. Screening for intimate partner violence in health care settings: $\mathrm{A}$ randomized trial. JAMA 2009;302(5):493-501. [http://dx.doi.org/10.1001/jama.2009.1089]
Jewkes R. Intimate partner violence: The end of routine screening. Lancet 2013;382(9888):190-191. [http:// dx.doi.org/10.1016/S0140-6736(13)60584-X]

8. Joyner K, Mash B. A comprehensive model for intimate partner violence in South African primary care Action research. BMC Health Serv Res 2012;12:399. [http://dx.doi.org/10.1186/1472-6963-12-399]

. Feder G, Davies RA, Baird K, et al. Identification and referral to improve safety (IRIS) of women experiencing domestic violence with a primary care training and support programme: A cluster randomised controlled trial. Lancet 2011;378(9805):1788-1795. [http://dx.doi.org/10.1016/S0140-
$6736(11) 61179-3$ ]

Robinson L, Spilsbury K. Systematic review of the perceptions and experiences of accessing health services
by adult victims of domestic violence. Health Soc Care Con org/10.1111/j.1365-2524.2007.00721.x]

1. Narula A, Agarwal G, McCarthy L. Intimate partner violence: Patients' experiences and perceptions in family practice. Fam Pract 2012;29(5):593-600.

Chang JC, Cluss PA, Ranieri L, et al. Health care interventions for intimate partner violence: What women want. Womens Health Issues 2005;15(1):21-30

13. Colombini M, Mayhew SH, Ali SH, Shuib R, Watts C. An integrated health sector response to violence gainst women in Malaysia: Lessons for supporting scale up. BMC Public Health 2012;12:548. [http://

Goicolea I, Briones-Vozmediano E, Ohman A, Edin K, Minvielle F, Vives-Cases C. Mapping and exploring
health systems' response to intimate partner violence in SP dx.doi.org/10.1186/1471-2458-13-1162]

15. Jacobs T, Jewkes R. Vezimfilho : A model for health sector response to gender violence in South Africa. Int J Gynecol Obstet 2002;78(Suppl 1):51-56.

S Afr Med J 2014;104(8):556-557. DOI:10.7196/SAMJ.8511 